\title{
REFEREES VOLUME 64
}

The following are thanked for the time and effort they have given to providing reviews of manuscripts submitted for publication in the last 12 months:

\begin{tabular}{|c|c|}
\hline Abdioglu, Emel & Ece, Ömer Işık \\
\hline Alba, Maria D. & Feng, Xionghan \\
\hline Alves, Marcelo E. & Ferrage, Eric \\
\hline Andreola, Fernanda & Frost, Ray L. \\
\hline Aristilde, Ludmilla & Garcia, Nelson Juan \\
\hline Assemi, Shoeleh & García Rodríguez, Juan \\
\hline Austin, Jay C. & Gimeno, Domingo \\
\hline Awwiller, David & Glezakou, Vanda \\
\hline Balan, Etienne & Gräsle, Werner \\
\hline Bauluz, Blanca & Greathouse, Jeffery A. \\
\hline Bizovská, Valéria & Greenwell, Chris \\
\hline Boek, Edo & Hanlie, Hong \\
\hline Bortoluzzi, Edson & He, Hongping \\
\hline Bozkaya, Ömer & Heinz, Hendrik \\
\hline Brendlé-Miehé, Jocelyne & Hillier, Steve \\
\hline Brigatti, Maria Franca & Holmboe, Michael \\
\hline Cameselle, Claudio & Hubert, Fabien \\
\hline Carretero, Maria Isabel & Huff, Warren D. \\
\hline Celik Karakaya, Muazzez & Huggett, Jennifer \\
\hline Cervini-Silva, Javiera & Iborra, Cesar Viseras \\
\hline Chatel, Gregory & Ilton, Eugene \\
\hline Chen, Zongyuan & Jaber, M. \\
\hline Cheshire, Michael C. & Jaisi, Deb P. \\
\hline Chrissopoulou, Kiriaki & Janek, Marian \\
\hline Christidis, George & Johnston, Cliff, T. \\
\hline Ciftci, Emin & Kaufhold, Stephan \\
\hline Cobas Roade Marta & Keeling, John \\
\hline Cox, Julia & Keller, Lukas \\
\hline Cuadros, Javier & Kocaoba, Sevgi \\
\hline Dahrazma, Behnaz & Komadel, Peter \\
\hline Darder, Margarita & Kremleva, Alena \\
\hline De Las Heras Cisa, F. & Kristof, J. \\
\hline Xavier & Kumpulainen, Sirpa \\
\hline Deneele, Dimitri & Kuznicki, Tetyana \\
\hline Derkowski, Arkadiusz & Legg, Benjamin \\
\hline Detellier, Christian & Li, Zhiqing \\
\hline Dumon, Mathijs Alain & Li, Guoxin \\
\hline Henri & Li, Zhaohui \\
\hline
\end{tabular}

Abdioglu, Emel

Alves, Marcelo E.

Andreola, Fernanda

Aristilde, Ludmilla

Assemi, Shoele

Awwiller, David

Balan, Etienne

Bauluz, Blanca

Boek, Edo

Bortoluzzi, Edson

aya, Omer

Brigatti, Maria Franca

Cameselle, Claudio

Carretero, Maria Isabel

Celik Karakaya, Muazzez

Chatel, Gregory

Chen, Zongyuan

Cheshire, Michael C.

Chrissopoulou, Kiriak

Christidis, George

Cox, Julia

Cuadros, Javier

Dahrazma, Behnaz

arder, Margarita
Xavier
Deneele, Dimitri
Derkowski, Arkadiusz Henri

\author{
Ece, Ömer Iş1k \\ eng, Xionghan \\ , Eric \\ Garcia, Nelson Juan \\ Gimeno, Domingo \\ Glezakou, Vanda \\ Greathouse, Jeffery A. \\ Greenwell, Chris \\ Heinz, Hendrik \\ Hillier, Steve \\ Holmboe, Michae \\ Huggett, Jennifer \\ Iborra, Cesar Viseras \\ Jaber, M. \\ Jaisi, Deb P. \\ Janek, Marian \\ Keeling, John \\ Keller, Lukas \\ Kremleva, Alena \\ Kristof, J. \\ Legg, Benjamin \\ Zhiqing \\ Li, Zhaohui
}

Liu, Jiang-Feng

Liu, Cheng-hua

Loganathan, Narasimhan

López-Galindo, Alberto

Lorenz, Christian

Lu, Xiancai

Luetzenkirchen, Johannes

Mako, Eva

Mareschal, Louis

Marry, Virginie

Mastrocicco, M.

McAdam, Maggie, M.

McBriarty, Martin

McCarty, Douglas

Meier, Lorenz

Meunier, Alain

Mussel, Wagner da Nova

Nascimento, Ronaldo

Ngouana, Brice

Nieto, Carlos

Nzengung, Valentine

Olin, Markus

Onal, Muserref

Orakdogen, Nermin

Osacky, Marek

Paineau, Erwan

Paipetis, Alkiviadis

Papoulis, Dimitrios

Pingxiao, Wu

Pizarro, Jaime

Pozo Rodriguez, Manuel

Prakash, K.

Rampe, Elizabeth

Reich, Tobias

Robinson, R.G.

Roush, Ted

Ryan, Peter

Sarkar, Binoy
Sasamoto, Hiroshi

Sassi, Michel

Sawada, James A. Schampera, Birgit

Schanz, Tom

Schroeder, Paul

Sellin, Patrik

Siddiqua, Sumi

Simić, Aleksandar

Skiba, Michal

Solling, Theis

Stegailov, Vladimir

Suarez, Mercedes

Szczerba, Marek

Szilagyi, Robert

Tao, Qi

Tateo, Fabio

Thomas, Sabu

Thompson, Aaron

Thyagaraj, T.

Triantafyllidis, Kostas

Tunega, Daniel

Türkmenoğlu, Asuman G.

Ufer, Kristian

Vicente, Miguel A.

Villar, M.V.

Wang, Jianwei

Wersin, Paul

Wilson, M. Jeff

Xiong, Yingqian

Yalçın, Hüseyin

Zagórski, Zbigniew

Zhang, Z.Z.

Zumsteg, Rolf 